Available online at
Elsevier Masson France

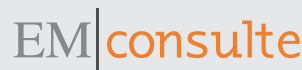

www.em-consulte.com/en

\title{
Eugenia uniflora fruit (red type) standardized extract: a potential pharmacological tool to diet-induced metabolic syndrome damage management
}

\author{
Pathise Souto Oliveira ${ }^{\mathrm{a}, 1}$, Vitor Clasen Chaves ${ }^{\mathrm{b}, 1}$, Natália Pontes Bona ${ }^{\mathrm{a}}$, \\ Mayara Sandrielly Pereira Soares ${ }^{c}$, Juliane de Souza Cardoso ${ }^{a}$, Flávia Aleixo Vasconcellos ${ }^{\mathrm{d}}$, \\ Rejane Giacomelli Tavares ${ }^{\mathrm{a}}$, Marcia Vizzotto ${ }^{\mathrm{e}}$, Luísa Mariano Cerqueira da Silva ${ }^{\mathrm{f}}$, \\ Fabiane Borelli Grecco ${ }^{f}$, Giovana Duzzo Gamaroc, Roselia Maria Spanevelloc, \\ Claiton Leoneti Lencina ${ }^{a}$, Flávio Henrique Reginatto ${ }^{\mathrm{b}, * *}$, Francieli Moro Stefanello ${ }^{\mathrm{a}, *}$ \\ a Laboratório de Biomarcadores, Centro de Ciências Químicas, Farmacêuticas e de Alimentos, Universidade Federal de Pelotas, Campus Universitário s/n, \\ Pelotas, RS, Brazil \\ b Laboratório de Farmacognosia, Programa de Pós-Graduação em Biotecnologia e Biociências, Universidade Federal de Santa Catarina, Florianópolis, SC, Brazil \\ ${ }^{\mathrm{c}}$ Laboratório de Neuroquímica, Inflamação e Câncer, Centro de Ciências Químicas, Farmacêuticas e de Alimentos, Universidade Federal de Pelotas, Campus \\ Universitário s/n, Pelotas, RS, Brazil \\ d Laboratório de Química Aplicada a Bioativos, Centro de Ciências Químicas, Farmacêuticas e de Alimentos, Universidade Federal de Pelotas, Campus \\ Universitário s/n, Pelotas, RS, Brazil \\ e Empresa Brasileira de Pesquisa Agropecuária, Centro de Pesquisa Agropecuária de Clima Temperado, Pelotas, RS, Brazil \\ f Laboratório de Patologia Animal, Programa de Pós-Graduação em Veterinária, Universidade Federal de Pelotas, Campus Universitário s/n, Pelotas, RS, Brazil
}

\section{A R T I C L E I N F O}

Article history:

Received 13 March 2017

Received in revised form 22 May 2017

Accepted 28 May 2017

\section{Keywords:}

Metabolic syndrome

Eugenia uniflora

Phenolic compounds

Pharmacological management

\section{A B S T R A C T}

The aim of this study was to investigate the effect of Eugenia uniflora fruit (red type) extract on metabolic status, as well as on neurochemical and behavioral parameters in an animal model of metabolic syndrome induced by a highly palatable diet (HPD). Rats were treated for 150 days and divided into 4 experimental groups: standard chow (SC) and water orally, SC and E. uniflora extract $(200 \mathrm{mg} / \mathrm{kg}$ daily, p. o), HPD and water orally, HPD and extract. Our data showed that HPD caused glucose intolerance, increased visceral fat, weight gain, as well as serum glucose, triacylglycerol, total cholesterol and LDL cholesterol; however, E. uniflora prevented these alterations. The extract decreased lipid peroxidation and prevented the reduction of superoxide dismutase and catalase activities in the prefrontal cortex, hippocampus and striatum of animals submitted to HPD. We observed a HPD-induced reduction of thiol content in these cerebral structures. The extract prevented increased acetylcholinesterase activity in the prefrontal cortex caused by HPD and the increase in immobility time observed in the forced swim test. Regarding chemical composition, LC/MS analysis showed the presence of nine anthocyanins as the major compounds. In conclusion, E. uniflora extract showed benefits against metabolic alterations caused by HPD, as well as exhibited antioxidant and antidepressant-like effects.

(c) 2017 Published by Elsevier Masson SAS.

\footnotetext{
* Corresponding author at: Universidade Federal de Pelotas, Campus Universitário s/n, CEP 96160-000, Capão do Leão, RS, Brazil.

** Corresponding author at: Universidade Federal de Santa Catarina, Florianópolis, SC, Brazil.

E-mail addresses: flavio.reginatto@ufsc.br (F.H. Reginatto),

francieli.stefanelo@ufpel.edu.br (F.M. Stefanello).

1 These authors equally contributed to this work.
}

\section{Introduction}

Metabolic syndrome (MetS) is a major health problem, which predisposes to the development of type 2 diabetes, cardiovascular and kidney diseases [1]. It is characterized by the presence of three or more of the following risk factors: hypertension, hyperglycemia, dyslipidemia, obesity and insulin resistance (IR) [1]. The prevalence of MetS is rapidly increasing worldwide including in developing countries, which is due primarily to prevailing sedentary lifestyles and unhealthy eating habits [2]. 
Experimental studies in animal models have shown that chronic consumption of diets with a high carbohydrate and fats content mimics the main signs of MetS in humans, especially dyslipidemia, hyperglycemia, glucose intolerance, obesity and IR [3]. Moreover, the consumption of these diets may increase fat deposits and free fatty acid (FFA) levels in the tissues and circulation leading to the development of a pro-inflammatory and pro-oxidant state [4].

It is known that the increase of reactive oxygen species (ROS) as well as pro-inflammatory cytokines is directly related to the development of MetS [5]. Moreover acetylcholinesterase (AChE) may contribute to the pathways controlling inflammatory and immune responses [6] and some reports demonstrated that cholinesterase activities were altered in diabetes and MetS [7]. In addition, there is a growing appreciation that the complications of MetS and obesity may result in increased risk for neurological co-morbidities like depressive illness [8]. In this context, studies indicate that oxidative stress mediates the neuropathological processes in a series of neurodegenerative diseases, and neuropsychiatric disorders [9].

Considering the variety of factors involved in the development of MetS, the search for potential new treatments still remains relevant. In this way, epidemiological studies have suggested an inverse relation between the consumption of polyphenol-rich foods and the risk of degenerative diseases. Therefore, there has been a great deal of interest in the screening and characterization of novel potentially therapeutic compounds of polyphenol-rich extracts obtained from foods and medicinal plants [10].

Eugenia uniflora (Myrtaceae) is widely distributed throughout South America [11]. Studies with its leaf extracts have shown numerous actions such as antioxidant and anti-inflammatory effects, reduction of triacylglycerol (TAG), reduced weight gain and decreased glucose levels in the blood [11,12]. However, the lack of studies related to the pharmacological properties of $E$. uniflora fruits, despite the presence of anthocyanins, polyphenols as major chemical constituents, make this fruit a source of antioxidant compounds, which are desirable to the patients with MetS [13]. In this sense, this work aimed at evaluating the effects of E. uniflora fruit (red type) extracts on metabolic status, as well as on neurochemical and behavioral parameters in rats fed with a highly palatable diet (HPD).

\section{Materials and methods}

\subsection{Phytochemicals}

\subsubsection{Extraction}

E. uniflora fruits (red type) were harvested in an orchard belonging to Embrapa Clima Temperado (Brazilian Agricultural Research Corporation) Pelotas/RS, Brazil (31 $40^{\prime} 47^{\prime \prime S}$ and $\left.52^{\circ} 26^{\prime} 24^{\prime \prime} \mathrm{W}\right)$. After picking, the fruits were immediately frozen at $-20^{\circ} \mathrm{C}$ and protected from light. The extracts were prepared according to Bordignon et al. [14] with modifications. Briefly, unprocessed frozen E. uniflora fruits $(30 \mathrm{~g})$ were sonicated for $30 \mathrm{~min}$ at $25^{\circ} \mathrm{C}$ in $90 \mathrm{~mL} 70: 30 \mathrm{v} / \mathrm{v}$ ethanol-water ( $\mathrm{pH} \mathrm{1.0)}$. The crude extracts were filtered; the ethanol removed under reduced pressure and then lyophilized.

\subsubsection{Total phenolic, flavonoid and anthocyanin contents}

The total phenolic content was determined according to Singleton et al., [15] with minor modifications and expressed as milligrams of gallic acid per $1 \mathrm{~g}$ of dried extract. The total flavonoid content was determined as described by Miliauskas et al., [16] and results were expressed as milligrams of gallic acid per $1 \mathrm{~g}$ of dried extract. Anthocyanins were quantified by the $\mathrm{pH}$ differential method [17] and reported as milligrams of cyanidin-3-glucoside per $1 \mathrm{~g}$ of dried extract. Data were expressed as mean \pm SD and all analyses were performed in triplicate.

\subsubsection{Identification of anthocyanins by LC/PDA/MS/MS}

Anthocyanins were identified using an Acquity-UPLC ${ }^{\mathrm{TM}}$ coupled to a photodiode array detector (PDA) and to a high-resolution mass spectrometer $\left(\mathrm{Xevo}^{\mathbb{R}}\right.$ G2 QTof model - WATERS ${ }^{\circledR}$ ). The chromatographic separation was performed using a C18 chromatography column (Synergi ${ }^{\mathrm{TM}}$ - Phenomenex ${ }^{\mathbb{R}}$ ) $4 \mu \mathrm{m}, 150 \times 2.0 \mathrm{~mm}$ at $40^{\circ} \mathrm{C}$, and the injection volume was $5 \mu \mathrm{L}$. The elution was carried out using an aqueous solution of formic acid $2 \%$ (solvent $\mathrm{A}$ ) and acetonitrile with $1 \%$ of formic acid (solvent $B$ ). The method used a linear gradient at constant flow $\left(0.4 \mathrm{~mL} \mathrm{~min}^{-1}\right)$. The total time of analysis was $34 \mathrm{~min}$ according to the following conditions: 0 $10 \mathrm{~min}, 5-12 \%$ B; $10-29 \mathrm{~min}, 12-18 \% \mathrm{~B} ; 29-33 \mathrm{~min}, 18 \% \mathrm{~B}$; $33-$ $34 \mathrm{~min}, 5 \% \mathrm{~B}$. Detection was performed at $520 \mathrm{~nm}$, and the range of spectral scanning in the visible region was $450-600 \mathrm{~nm}$ (PDA). Mass spectrometry detection was carried out with an electrospray ionization source (ESI) set on negative ion mode, capillary voltage, $1.0 \mathrm{kV}$, source block temperature, $120^{\circ} \mathrm{C}$, desolvation temperature, $600^{\circ} \mathrm{C}$; nebulizer nitrogen flow rate, $80 \mathrm{~L} \mathrm{~h}^{-1}$, desolvation nitrogen gas flow, $800 \mathrm{~L} \mathrm{~h}^{-1}$, and cone voltage, $40 \mathrm{~V}$, controlled by MassLynx v.4.1 software for data acquisition and processing. The mass scanning ranged from $\mathrm{m} / \mathrm{z} 200$ to 1500 with a scan time of $0.5 \mathrm{~s}$. MS/MS analysis was performed using a collision energy ramp (10$30 \mathrm{eV})$; and with argon as collision gas. All analyses were performed in triplicate.

\subsection{Animals and drug treatments}

Male wistar rats aged 21 days maintained at $21-25^{\circ} \mathrm{C}$ with free access to water and food, under a 12:12 h light:dark cycle were used throughout this study

Rats were divided into four groups: (1) standard chow (SC) group + vehicle, which received standard laboratory rat chow (50\% carbohydrate, from starch, $22 \%$ protein and $4 \%$ fat) and water orally; (2) SC + E. uniflora, which received standard chow and $200 \mathrm{mg} / \mathrm{Kg}$ /day of E. uniflora orally; (3) highly palatable diet (HPD) group + vehicle, which received an enriched sucrose diet (65\% carbohydrates $34 \%$ being from condensed milk, $8 \%$ from sucrose and $23 \%$ from starch, $25 \%$ protein and $10 \%$ of fat) and water orally (4) HPD + E. uniflora, $200 \mathrm{mg} / \mathrm{kg} /$ day orally. The HPD and dose of $E$. uniflora extract used in this study were chosen according to Oliveira et al., [18]. The experiments were performed after the approval of the local Ethics Committee (CEEA no. 9125) and no efforts were spared to minimize animal suffering.

\subsection{Body weight gain and food intake}

Changes in body weight and food intake patterns of rats were measured throughout the experimental period. The weight of each rat was recorded on day 0 and at weekly intervals throughout the course of the study. The quantity of food consumed by each group was recorded weekly, and the food consumption per rat was calculated for all groups.

\subsection{Sample collection and biochemical assay}

After 150 days of food and extract administration and $24 \mathrm{~h}$ after the last behavioral test, the animals were euthanized by decapitation having fasted for $6 \mathrm{~h}$. At euthanasia, visceral fat was weighed and the blood collected. Serum was obtained by centrifugation at $4000 \mathrm{rpm}\left(4^{\circ} \mathrm{C}\right)$ for $15 \mathrm{~min}$. Liver and adipose tissue were collected for further histopathological analysis. Prefrontal cortex, hippocampus and striatum were collected and stored at $-80^{\circ} \mathrm{C}$ for subsequent biochemical analyses. 


\subsubsection{Glucose tolerance test}

Rats were injected intraperitoneally with a $50 \%$ glucose solution load of $2 \mathrm{mg} / \mathrm{g}$ of body weight. The glucose levels for all the groups were estimated by the glucometer (AccuChek Active, Roche Diagnostics ${ }^{\circledR}$, USA) at 30,60 , and 120 min after the injection by a small tail puncture.

\subsubsection{Serum biochemical parameters}

Measurements of serum glucose, total cholesterol, cholesterolLDL, cholesterol-HDL, TAG, urea, uric acid and alanine aminotransferase (ALT) levels were determined using commercially available diagnostic kits supplied by Labtest ${ }^{\mathbb{R}}$ (Labtest, MG, Brazil).

\subsubsection{Histopathological analysis}

Liver and adipose tissue samples were fixed in $10 \%$ buffered formalin ( $\mathrm{pH} 7.4$ ) and embedded in paraffin, cleaved and routinely processed. Samples were cut into 5 - $\mu \mathrm{m}$ sections and stained with hematoxylin and eosin. Histological analyses were performed in all groups.

\subsection{Behavioral analysis}

\subsubsection{Forced swim test (FST)}

The depressive-like behavior was evaluated by the total duration of immobility in the FST, as previously described by Kaster et al., [19]. Rats were individually forced to swim in an open cylindrical container, with water at $25 \pm 1{ }^{\circ} \mathrm{C}$ and the total duration of immobility during a 5 min period was scored: rats were judged to be immobile when they ceased struggling and remained floating motionless in the water, making only those movements necessary to keep their head above water.

\subsubsection{Open-field test}

The ambulatory behavior was assessed in an open-field test as previously described by Kaster et al., [20]. The apparatus consisted of a box with the floor of the arena divided into 12 equal squares and placed in a sound-free room. Animals were placed in the rear left square and left to freely explore it for 5 min during which time the number of squares crossed with all paws (crossing) was counted.

\subsubsection{Elevated plus-maze}

Anxiety-related behaviors were evaluated using the elevated-plus maze (EPM). Animals were placed in the center of the EPM and were free to explore for $5 \mathrm{~min}$. The number of entries into the open and closed arms, aswellas time spentin the arms was measured during the 5-min test. To evaluate the animal's anxiety we used an index calculated with the following equation. Anxiety index values range from 0 to 1, with a higher value indicating increased anxiety [21].

Anxiety index $=1-[$ (open arm time $/ 5 \mathrm{~min})+($ open arm entry $/$ total entry)]/2

\subsection{Tissue preparation}

Prefrontal cortex, hippocampus and striatum were homogenized in sodium phosphate buffer $\mathrm{pH} 7.4$ containing $\mathrm{KCl}$. The homogenates were centrifuged at $3500 \mathrm{rpm}$ for $10 \mathrm{~min}$ at $4{ }^{\circ} \mathrm{C}$ and the supernatant was separated and used for neurochemical analyzes. Protein was determined by the method of Lowry et al., [22] or Bradford [23].

\subsection{Oxidative stress parameters}

\subsubsection{Thiobarbituric acid-reactive substances (TBARS)}

TBARS levels were determined according to the method described by Ohkawa et al., [24] and reported as nmol TBARS/ mg protein.

\subsubsection{Total thiol content assay}

This assay was performed as described by Aksenov and Markesbery [25]. Results were reported as nmol TNB/mg of protein.

\subsubsection{Antioxidant enzyme activities}

Catalase (CAT) activity was assayed according to Aebi [26] based on the decomposition of $\mathrm{H}_{2} \mathrm{O}_{2}$. Superoxide dismutase (SOD) activity was measured by the method described by Misra and Fridovich [27] that is based on the inhibition of superoxide dependent adrenaline auto-oxidation. Glutathione peroxidase (GPX) activity was measured using commercially available diagnostic kits supplied by RANDOX (Brazil). The enzyme activities were reported as units/mg protein.

\subsection{Acetylcholinesterase (AChE) activity}

AChE activity was determined as described by the colorimetric method of Ellman et al., [28] with modification and the enzyme activity was expressed as $\mu \mathrm{mol} / \mathrm{h} / \mathrm{mg}$ of protein.

\subsection{Statistical analysis}

The values were expressed as mean \pm S.E.M. Glucose tolerance was analyzed by repeated measures ANOVA and Bonferroni's posthoc test. Parametric variables were tested by two-way ANOVA and Bonferroni post-hoc test. A value of $P \leq 0.05$ was considered to be significant. Analyses were performed using the GraphPad PRISM $5^{\circledR}$ software.

\section{Results}

\subsection{Phytochemical characterization}

The total phenolic content was $7.92 \pm 0.23 \mathrm{mg} / \mathrm{g}$ of dried extract while the total flavonoid content, $5.50 \pm 0.68 \mathrm{mg} / \mathrm{g}$ of dried extract

Table 1

Chromatographic and spectroscopic profile of anthocyanins from fruits of E. uniflora (red type).

\begin{tabular}{|c|c|c|c|c|c|}
\hline $\mathrm{RT}(\min )$ & $\lambda_{\max }(\mathrm{nm})$ & {$[\mathrm{M}+\mathrm{H}]^{+}(m / z)$} & Error (ppm) & MS/MS $(m / z)$ & Identification $^{\mathrm{a}}$ \\
\hline 6.50 & 523 & 465,1004 & $-6,2$ & 303 & Delphinidin-O-glucoside \\
\hline 7.24 & 515 & 449,1073 & $-2,4$ & 287 & Cyanidin-3-O-glucoside ${ }^{a}$ \\
\hline 8.27 & 515 & 449,1065 & $-4,2$ & 287 & Cyanidin-O-galactoside \\
\hline 8.68 & 520 & 479,1166 & $-5,0$ & 317 & Petunidin- $O$-hexoside \\
\hline 10.07 & 505 & 433,1130 & 1,2 & 271 & Pelargonidin-3-O-glucoside ${ }^{a}$ \\
\hline 10.87 & 510 & 579,1751 & 6,4 & 271 & Pelargonidin- $\mathrm{O}$-rutinoside \\
\hline 11.58 & 523 & 493,1331 & $-3,0$ & 331 & Malvidin-3-O-glucoside ${ }^{a}$ \\
\hline 13.84 & 523 & 463,1221 & $-4,1$ & 331 & Malvidin- $O$-pentoside \\
\hline 20.17 & 525 & 535,1471 & 3,6 & 331 & Malvidin-O-acetylhexoside \\
\hline
\end{tabular}

${ }^{a}$ Identified by injection of corresponding authentic standards, literature data and mass spectra database (Reaxys $\left.{ }^{\mathbb{R}}\right)$. 
was detected. Total monomeric anthocyanin content was1.72 $\pm 0.05 \mathrm{mg} / \mathrm{g}$ of dried extract.

Analysis by LC/PDA/MS/MS showed the presence of nine anthocyanins (Table 1 ) of which just delphinidin- $O$-glucoside [29] and cyanidin-3-O-glucoside [29,30] were previously described for E. uniflora fruits. Considering the intensity of the peaks, it was possible to describe cyanidin-3-O-glucoside as the major anthocyanin present in these fruits. Furthermore, as far as we are aware, this is the first report of cyanidin- $O$-galactoside, petunidin$O$-hexoside, pelargonidin-3-O-glucoside, pelargonidin-O-rutinoside, malvidin-3-O-glucoside, malvidin-O-pentoside, and malvidin-O-acetylhexoside in E. uniflora fruits.

\subsection{Metabolic status}

As shown in Fig. 1, HPD induced an impaired glucose tolerance $(P<0.01)$ and treatment with $E$. uniflora was able to prevent this change in the animals submitted to HPD.

Table 2 shows that $E$. uniflora treatment prevented the increase of visceral fat mass (treatment: $[\mathrm{F}(1,27)=7.40 ; P<0.05]$, HPD: [F $(1,27)=32.34, P<0.001]$, interaction: $[F(1,27)=4.41 ; P<0.05])$, blood glucose levels (E. uniflora: $[\mathrm{F}(1,18)=8.95 ; P<0.05]$, HPD: $[\mathrm{F}(1,18)=14.38 ; P<0.001]$, interaction: $[\mathrm{F}(1,18)=24.63 ; P<0.001])$, total cholesterol (treatment: $[\mathrm{F}(1,22)=28.43 ; P=0.001]$, HPD: [F $(1,22)=36.94 ; P<0.001]$, interaction: $[F(1,22)=24.35 ; P<0.001])$, cholesterol-LDL (treatment: $[\mathrm{F}(1,14)=44.10 ; P<0.001]$, HPD: [F $(1,14)=41.52 ; P<0.001]$, interaction: $[\mathrm{F}(1,14)=28.11 ; P<0.001])$, TAG (treatment: $[\mathrm{F}(1,13)=16.72 ; P<0.001]$, HPD $[\mathrm{F}(1,13)=6.85$; $P<0.05]$, interaction: $[F(1,13)=6.11 ; P<0.05])$ caused by HPD. Although we did not find an interaction between groups, E. uniflora consumption was able to prevent the increase in the weight gain caused by HPD (treatment: $[\mathrm{F}(1,25)=6.60 ; P<0.05]$, HPD [F $(1,25)=23.44 ; P<0.001]$, interaction: $[F(1,25)=3.48 ; P \geq 0.05])$. However, there was no significant difference in any of the groups tested as to cholesterol-HDL, urea, uric acid and ALT $(P>0.05)$.

Regarding histopathological analyses, we did not observe degenerative, necrotic or inflammatory processes in liver and adipose tissue in any of the tested groups (data not shown).

\subsection{Behavioral parameters}

The rats submitted to HPD protocol exhibited a significant increase in immobility time in the FST when compared to control animals, suggesting a depressive-like state. Still, the treatment with E. uniflora extract prevented this increase caused by HPD, suggesting an antidepressant-like effect (Fig. 2B) (treatment: $[F$ $(1,24)=3.77, P>0.05]$, HPD: $[F(1,24)=9.63, P<0.05]$, interaction: $[F$ $(1,24)=8.52, P<0.05])$.

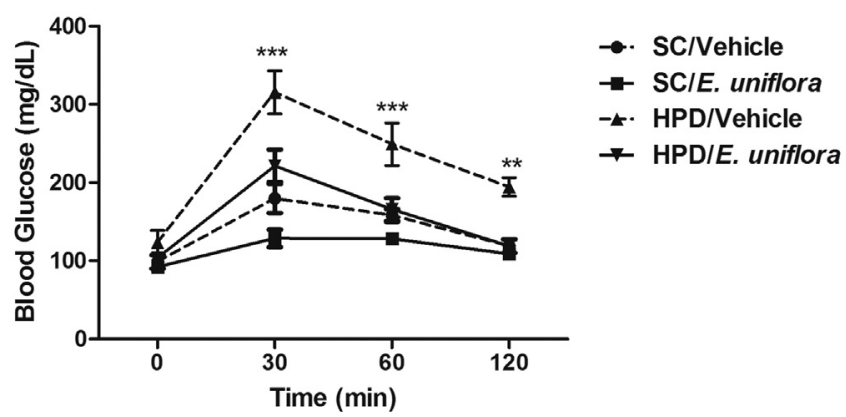

Fig. 1. Glucose tolerance test measured at the baseline (0), 30, 60 and $120 \mathrm{~min}$ after glucose injection $\left(2 \mathrm{mg} / \mathrm{g}\right.$ body weight). Data are expressed as mean \pm S.E.M. $\left({ }^{* * *}\right)$ $P<0.001$ compared to the vehicle/SC. (**) $P<0.01$ compared to the vehicle/SC. Repeated measures ANOVA followed by Bonferroni post-hoc test. SC=Standard Chow; HPD = Highly Palatable Diet.
In order to rule out nonspecific motor effects that could interfere in the FST, rats were also submitted to the open-field test (Fig. 2A) and no significant change in the ambulatory behavior was observed in the open-field test (treatment: $[F(1,23)=0.76, P>0.05]$, HPD: $[F(1,23)=1.11, P>0.05]$, interaction: $[F(1,23)=0.64, P>0.05])$.

We also observed that there was no difference in the anxiogenic profile of animals in any of the groups tested (anxiety index) (treatment: $[F(1,24)=2.51, P>0.05]$, HPD: $[F(1,24)=0.43, P>0.05]$, interaction: $[F(1,24)=0.67, P>0.05]$ ) (data not shown).

\subsection{Neurochemical parameters}

Table 3 shows that although there is no interaction between groups, E. uniflora treatment was able to prevent the increase of TBARS levels in hippocampus of the animals submitted to HPD. In addition, E. uniflora per se reduced TBARS levels when compared to control group (treatment: $[F(1,24)=18.03, P<0.001]$, HPD: $[F$ $(1,24)=57.63, P<0.001]$, interaction: $[F(1,24)=0.0018, P>0.05])$. When thiol content was evaluated no interaction between treatment and diet was observed, but it was possible to note a significant decrease in this parameter by HPD (treatment: $[F$ $(1,21)=0.29 P>0.05]$, HPD: $[F(1,21)=14.49, P<0.001]$, interaction: $[F(1,21)=1.27, P>0.05])$. As regard the activity of antioxidant enzymes, it was observed that the E. uniflora treatment prevented the reduction of SOD (treatment: $[F(1,22)=0.33, P>0.05]$, HPD: $[F$ $(1,22)=11.47, P<0.01]$, interaction: $[F(1,22)=16.28, P<0.001])$ and CAT caused by HPD consumption in the hippocampus (treatment: $[F(1,18)=0.275, P>0.05]$, HPD: $[F(1,18)=0.58, P>0.05]$, interaction: $[F(1,18)=23.52, P<0.001])$. GPX was not altered in any groups tested $(P>0.05)$.

Additionally, we evaluated the effects of E. uniflora extract on oxidative stress parameters in the prefrontal cortex (Table 3). The treatment with this extract also was able to prevent the increase in TBARS levels induced by HPD, as well as in the SC group (treatment: $[F(1,18)=5.06, \quad P<0.05], \quad$ HPD: $[F(1,18)=17.38, \quad P$ $<0.001]$, interaction: $[F(1,18)=10.86, P<0.05])$. Also, it was possible to observe a significant decrease in total thiol content induced by HPD (treatment: $[F(1,21)=0.45, P>0.05]$, HPD: $[F$ $(1,21)=4.63, P<0.05]$, interaction: $[F(1,21)=5.63, P<0.05])$.

In addition, E. uniflora treatment prevented the decreased activity of antioxidant enzymes such as SOD caused by consumption of HPD (treatment: $[F(1,17)=1.10, P>0.05]$, HPD: $[F$ $(1,17)=18.70, \quad P<0.001]$, interaction: $[F(1,17)=8.23, \quad P<0.05])$ and CAT (treatment: $[F(1,14)=1.39, \quad P>0.05], \quad$ HPD: $[F$ $(1,14)=18.30, P<0.001]$, interaction: $[F(1,14)=7.79, P<0.05])$. The antioxidant enzyme GPX showed no difference in the HPD or in the group treated with $E$. uniflora extract $(P>0.05)$.

Table 3 also shows the effects of $E$. uniflora extract on oxidative stress parameters in the striatum. It was observed that the extract was able to prevent the increase in TBARS levels induced by HPD, as well as in the SC group (treatment: $[F(1,28)=19.19, P<0.001]$, HPD: $[F(1,28)=6.83, P<0.05]$, interaction: $[F(1,28)=6.16, P<0.05])$. Besides, the thiol content was decreased by HPD (treatment: $[F$ $(1,18)=0.0011, P>0.05]$, HPD: $[F(1,18)=6.86, P<0.05]$, interaction: $[F(1,18)=11.82, \quad P<0.05]$ ) (Table 3 ). As regards antioxidant enzymes, $E$. uniflora treatment prevented the decreased activity of SOD caused by consumption of HPD (treatment: $[F(1,19)=4.68$, $P<0.05]$, HPD: $[F(1,19)=5.95, P<0.05]$, interaction: $[F(1,19)=8.19$, $P<0.05])$ and CAT (treatment: $[F(1,25)=2.60, P>0.05]$, HPD: $[F$ $(1,25)=6.63, P<0.05]$, interaction: $[F(1,25)=6.33, P<0.05])$. No significant difference in GPX activity was observed in any of the groups tested $(P>0.05)$.

As shown in Table 4,E. uniflora treatment prevented the increase in cortical AChE activity caused by consumption of HPD (treatment: $[F(1,19)=20.24, P<0.001], \quad H P D:[F(1,19)=4.77, P$ $<0.05]$, interaction: $[F(1,19)=5.07, P<0.05])$. In contrast, this 
Table 2

Effect of E. uniflora extract treatment in rats exposed to a highly palatable diet on metabolic parameters.

\begin{tabular}{|c|c|c|c|c|}
\hline & SC/Vehicle & SC/E. uniflora & HPD/Vehicle & HPD/E. uniflora \\
\hline Weight gain (g) & $373.20 \pm 21.35$ & $362.57 \pm 10.64$ & $474.62 \pm 19.64^{* * *}$ & $407.56 \pm 7.47^{\# \#}$ \\
\hline Visceral fat mass ( $\mathrm{g}$ ) & $15.66 \pm 1.86$ & $13.85 \pm 0.93^{\# \# \#}$ & $38.33 \pm 4.92^{* * *}$ & $24.30 \pm 1.75^{\# \#}$ \\
\hline Glycemia (mg/dL) & $77.40 \pm 3.79$ & $88.98 \pm 3.48^{\# \# \#}$ & $128.86 \pm 6.79^{* * *}$ & $82.10 \pm 2.84^{\# \# \#}$ \\
\hline Total cholesterol (mg/dL) & $105.1 \pm 3.86$ & $104.1 \pm 4.34^{\# \# \#}$ & $156.8 \pm 5.72^{* * *}$ & $109.4 \pm 1.63^{\# \# \#}$ \\
\hline Cholesterol LDL (mg/dL) & $46.77 \pm 2.53$ & $40.67 \pm 7.77^{\# \# \#}$ & $100.3 \pm 2.83^{* * *}$ & $45.87 \pm 4.07^{\# \# \#}$ \\
\hline Cholesterol HDL (mg/dL) & $49.47 \pm 1.95$ & $55.00 \pm 3.55$ & $43.37 \pm 3.48$ & $51.05 \pm 2.52$ \\
\hline Triacylglycerol (mg/dL) & $65.89 \pm 10.20$ & $55.68 \pm 6.86^{\# \#}$ & $98.00 \pm 3.20^{*}$ & $56.60 \pm 3.66^{\# \#}$ \\
\hline Urea $(\mathrm{mg} / \mathrm{dL})$ & $69.99 \pm 3.88$ & $55.98 \pm 4.62$ & $56.02 \pm 2.48$ & $54.29 \pm 2.32$ \\
\hline Uric acid (mg/dL) & $1.01 \pm 0.09$ & $1.02 \pm 0.04$ & $0.75 \pm 0.12$ & $0.84 \pm 0.21$ \\
\hline $\operatorname{ALT}(\mathrm{U} / \mathrm{mL})$ & $39.96 \pm 0.17$ & $37.84 \pm 0.30$ & $38.13 \pm 1.74$ & $36.33 \pm 0.39$ \\
\hline
\end{tabular}

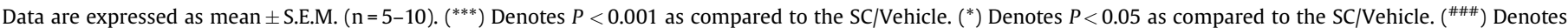
$P<0.001$ as compared to HPD/Vehicle. $\left({ }^{\# \#)}\right.$ Denotes $P<0.01$ as compared to HPD/Vehicle. SC $=$ Standard Chow; HPD $=$ Highly Palatable Diet.

enzyme activity was not altered in the hippocampus and striatum $(P>0.05)$.

\section{Discussion}

MetS is commonly associated with the development of IR and hypertension, increased glucose levels, TAG and LDL-cholesterol. Although the exact mechanisms that trigger this syndrome have not been well defined, visceral obesity and IR are the main factors involved [31]. On the other hand, dietary polyphenols constitute a large family of bioactive substances, which could be an effective nutritional strategy to improve the health of patients with MetS [32].

In the present study, we investigated the ability of $E$. uniflora fruit extract to prevent some metabolic, neurochemical and behavioral parameters. Our data showed that HPD-induced MetS caused glucose intolerance, increased weight and visceral fat, glucose, total cholesterol, LDL-cholesterol and TAG levels, and also

A

Open-field
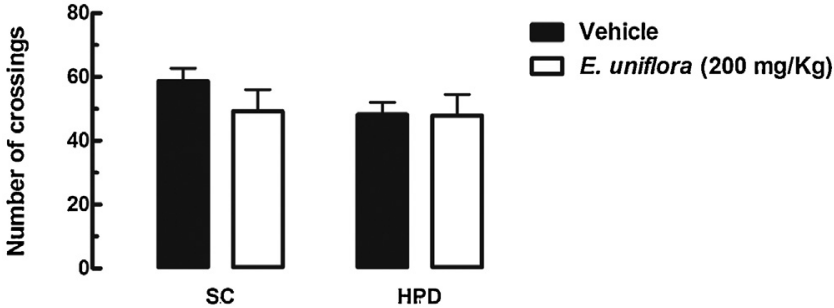

B

Forced Swimming Test
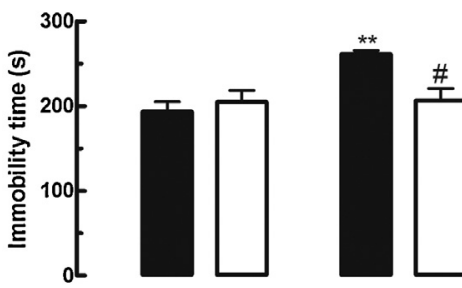

E. uniflora $(200 \mathrm{mg} / \mathrm{Kg})$

Fig. 2. Effect of $E$. uniflora extract treatment in rats exposed to a highly palatable diet on ambulatory behavior in the open-field test (A) and immobility time in the FST (B). The results are expressed as mean \pm S.E.M. $\left(\mathrm{n}=9-10\right.$ for group). ${ }^{* *} P<0.01$ when compared to the vehicle/SC. ${ }^{\#} P<0.05$ when compared to vehicle/HPD. Twoway ANOVA followed by Bonferroni post-hoc test. SC = Standard Chow; HPD = Highly Palatable Diet. induced behavioral and neurochemical alterations. Besides, we demonstrated that the changes caused by the consumption of HPD were prevented by E. uniflora extract. In addition, no histopathological changes were observed in liver and adipose tissue. The diet and treatment used did not alter the metabolism of hepatocytes or adipose cells, since they did not trigger any inflammatory or degenerative responses. In contrast, studies with high-fructose or high-fat diets demonstrated an increase in the size of adipocytes, as well as hepatic fat accumulation, fibrosis and inflammation in the liver [33-35].

Data in the literature suggest that E. uniflora fruits are a major source of phytochemicals, such as phenolic compounds, flavonoids and anthocyanins [36]. These compounds are known to present antioxidant, anti-inflammatory and neuroprotective properties, besides having beneficial effects on the metabolism of glucose and lipids [37]. It has been reported that anthocyanins, flavonoids and other phenolic compounds present important therapeutic effects in preventing obesity, type 2 diabetes, IR and dyslipidemia [38]. Although the mechanisms involved in these effects have not yet been elucidated, the main hypothesis of action of these chemical

Table 3

Effect of E. uniflora extract treatment in rats exposed to a highly palatable diet on oxidative stress parameters in the prefrontal cortex, hippocampus and striatum

\begin{tabular}{|c|c|c|c|c|}
\hline Parameters & SC/Vehicle & SC/E. uniflora & HPD/Vehicle & $\begin{array}{l}\text { HPD/E. } \\
\text { uniflora }\end{array}$ \\
\hline & \multicolumn{4}{|c|}{ Prefrontal cortex } \\
\hline TBARS & $0.99 \pm 0.13$ & $1.14 \pm 0.15^{\# \#}$ & $2.02 \pm 0.13^{* * *}$ & $1.26 \pm 0.13^{\# \#}$ \\
\hline $\begin{array}{l}\text { Total SH } \\
\text { content }\end{array}$ & $37.60 \pm 3.29$ & $32.52 \pm 3.01$ & $24.08 \pm 2.60^{*}$ & $33.17 \pm 2.81$ \\
\hline CAT & $1.41 \pm 0.10$ & $1.28 \pm 0.11^{\# \#}$ & $0.85 \pm 0.06^{* * *}$ & $1.16 \pm 0.01^{\#}$ \\
\hline SOD & $32.72 \pm 2.47$ & $29.07 \pm 2.95$ & $18.32 \pm 1.39^{* * *}$ & $26.16 \pm 1.35^{\#}$ \\
\hline \multirow[t]{2}{*}{ GPX } & $55.81 \pm 3.87$ & $53.83 \pm 3.07$ & $51.32 \pm 2.17$ & $50.26 \pm 3.37$ \\
\hline & \multicolumn{4}{|c|}{ Hippocampus } \\
\hline $\begin{array}{l}\text { Total SH } \\
\text { content }\end{array}$ & $30.04 \pm 2.35$ & $28.95 \pm 1.44$ & $20.87 \pm 0.95^{* *}$ & $23.97 \pm 0.35$ \\
\hline CAT & $1.51 \pm 0.12$ & $1.29 \pm 0.05$ & $1.12 \pm 0.05^{* *}$ & $1.57 \pm 0.06^{\# \# \#}$ \\
\hline SOD & $32.17 \pm 0.71$ & $26.57 \pm 1.60$ & $20.98 \pm 2.27^{* * *}$ & $27.62 \pm 0.64^{\#}$ \\
\hline \multirow[t]{2}{*}{ GPX } & $53.20 \pm 3.85$ & $51.90 \pm 3.05$ & $54.25 \pm 2.90$ & $65.32 \pm 2.50$ \\
\hline & \multicolumn{4}{|c|}{ Striatum } \\
\hline TBARS & $2.23 \pm 0.11$ & $1.76 \pm 0.25^{\# \# \#}$ & $3.50 \pm 0.31^{* * *}$ & $1.79 \pm 0.28^{\# \# \#}$ \\
\hline $\begin{array}{c}\text { Total SH } \\
\text { content }\end{array}$ & $34.28 \pm 0.93$ & $28.00 \pm 1.33$ & $23.11 \pm 1.26^{* *}$ & $29.51 \pm 2.41$ \\
\hline CAT & $1.82 \pm 0.17$ & $1.67 \pm 1.15$ & $1.09 \pm 0.08^{* *}$ & $1.68 \pm 0.16^{\#}$ \\
\hline SOD & $36.57 \pm 3.56$ & $34.56 \pm 3.34$ & $21.27 \pm 0.39^{* * *}$ & $35.78 \pm 2.36^{\#}$ \\
\hline GPX & $55.59 \pm 4.20$ & $66.92 \pm 2.21$ & $57.08 \pm 4.98$ & $58.73 \pm 2.53$ \\
\hline
\end{tabular}

Data are expressed as mean \pm S.E.M. $(n=5-10)$. TBARS levels are reported as nmol TBARS per mg protein, thiol content as nmol TNB per mg protein, enzyme activities (CAT, SOD, GPX) as units/mg protein. ( ${ }^{* * *}$ ) Denotes $P<0.001$ as compared to the SC/ Vehicle. $\left({ }^{* *}\right)$ Denotes $P<0.01$ as compared to the SC/Vehicle. $\left(^{*}\right)$ Denotes $P<0.05$ as compared to the SC/Vehicle. $\left(^{\# \# \#) ~} P<0.001\right.$ as compared to the HPD/Vehicle. $\left(^{\# \#)}\right.$ $P<0.01$ as compared to the HPD/Vehicle. $\left({ }^{\#}\right) P<0.05$ as compared to the HPD/ Vehicle. $\mathrm{SC}=$ Standard Chow; $\mathrm{HPD}=$ Highly Palatable Diet 
Table 4

Effect of $E$. uniflora extract treatment in rats exposed to a highly palatable diet on acetylcholinesterase activity in prefrontal cortex, hippocampus and striatum.

\begin{tabular}{lllll}
\hline & AChE $(\mu \mathrm{mol} / \mathrm{h} / \mathrm{mg}$ protein $)$ & \\
\cline { 2 - 5 } & SC/Vehicle & SC/E. uniflora & HPD/Vehicle & HPD/E. uniflora \\
\hline Prefrontal cortex & $1.24 \pm 0.118$ & $1.03 \pm 0.102$ & $1.66 \pm 0.091^{*}$ & $1.02 \pm 0.066^{\# \# \#}$ \\
Hippocampus & $1.25 \pm 0.012$ & $1.35 \pm 0.046$ & $1.23 \pm 0.087$ & $1.47 \pm 0.017$ \\
Striatum & $6.07 \pm 0.164$ & $6.03 \pm 0.010$ & $5.66 \pm 0.311$ & $5.17 \pm 0.195$ \\
\hline
\end{tabular}

Data are expressed as mean \pm S.E.M. $(\mathrm{n}=4-6)$. $\left({ }^{*}\right)$ Denotes $P<0.05$ as compared to the SC/Vehicle. $\left({ }^{\# \# \#) ~} P<0.001\right.$ as compared to HPD/Vehicle. SC = Standard Chow; HPD $=$ Highly Palatable Diet.

components of E. uniflora fruit extract in the prevention of IR and hyperglycemia may be attributed to the inhibition of carbohydrate digestion and absorption of glucose in the intestine, stimulation of insulin secretion by pancreatic beta cells, modulation of liver glucose secretion, activation of insulin receptors, glucose uptake in insulin sensitive tissues and modulating hepatic glucose production [37]. Manzano and Williamson [39] observed that fruit extracts containing phenolic acids, quercetin and tannin inhibited glucose transport in vitro via glucose active transporter-dependent sodium (SGLT1) and GLUT2 in membrane vesicles of the intestine. Hsu and Yen [40] demonstrated that supplementation of gallic acid reduced TAG, total cholesterol, LDL-cholesterol levels and visceral fat in mice fed a high fat diet. Regarding anthocyanins, it was reported that these compounds have very powerful healthpromoting effects and can be used as a functional food factor [41].

It is known that the polyphenols can play an important role in neutralizing ROS, acting to prevent pathologies related to oxidative stress such as MetS [12]. In this study, we demonstrated that the consumption of HPD increased TBARS levels and decreased the total thiol content in hippocampus, prefrontal cortex and striatum. However, E. uniflora treatment was able to prevent the increase in TBARS levels caused by HPD in all tissues tested. Corroborating our findings, Trevino and collaborators [42] also observed an increase in hippocampal lipid peroxidation in animals subjected to a MetS model induced by high calorie diet consumption. TBARS is a marker of lipid peroxidation formed by the reaction with cytotoxic products such as malondialdehyde released during the oxidation of unsaturated fatty acids and plays an important role in the complications of MetS [43]. Lipid peroxidation induced by the consumption of HPD may be associated with increased glucose levels, which can lead to increased production of ROS by glucose autoxidation, because chronic hyperglycemia leads to the formation of end product of advanced glycation damaging cells and stimulating the production of ROS [44]. Moreover, chronic hyperglycemia present in MetS can also contribute to neuronal damage and changes in thiol groups in the brain. In this study we observed that HPD decreased total thiol content in the brain structures tested, suggesting that this animal model induces protein damage to sulfhydryl groups. Cuhad and collaborators [45] also showed a decrease in total thiol content in cerebral cortex and hippocampus of diabetic rats with cognitive deficits.

Some studies have shown an association between decreased antioxidant defenses in brain structures and increased oxidative stress in MetS and type 2 diabetes [18,44]. Therefore, we evaluated the activity of antioxidant enzymes in the prefrontal cortex, striatum and hippocampus of animals submitted to HPD and treated with E. uniflora extract. Our data showed that the consumption of HPD significantly decreases the SOD and CAT activity in the brain structures tested; moreover, it was found that treatment with $E$. uniflora extract was able to prevent the reduction of the activity of these enzymes in these tissues. However, there was no significant difference in the activity of GPX enzyme in animal tissues from any of the groups tested.
It is well known that the CNS uses a significant amount of oxygen and ATP, and shows decreased antioxidant defenses compared with other tissues, resulting in a great susceptibility to oxidative stress [44]. It has also been shown that the high levels of ROS in MetS play an important role in the pathophysiology of depression [46]. In this study, HPD increased the immobility time in the FST indicating depressive-like behavior. However, treatment with E. uniflora extract prevented this alteration, suggesting an antidepressant-like effect. According to our findings, studies using animal models of depression have showed an increase in lipid peroxidation and decrease of antioxidant enzymes in the prefrontal cortex, striatum and hippocampus, suggesting that oxidative stress may play an important role in the relationship between MetS and depression since these structures are related to mood disorders, especially depression $[18,47]$. This hypothesis can be related to the antidepressant-like effect of the treatment with $E$. uniflora extract since it showed significant antioxidant action.

There is a large amount of data in the literature demonstrating that changes in the activity of AChE respond to various insults including oxidative stress and are related to the pathogenesis of a variety of diseases such as diabetes, inflammation, neurological disorders [7]. Therefore, we demonstrated that the HPD increased AChE activity in the prefrontal cortex; however, treatment with $E$. uniflora extract was able to prevent the increased activity of this enzyme in this tissue demonstrating a neuroprotective effect. Corroborating these findings, Zarros and collaborators [7] demonstrated a significant increase in rat brain AChE activity after the induction of diabetes. Based on this, we suggest that the cholinergic dysfunction present in diabetes and MetS may be related to cognitive and neurochemical dysfunctions, however the role of $\mathrm{AChE}$ in depression is still little explored and controversial [7]. Furthermore, phytochemicals present in E. uniflora extract could contribute to a modulation of the levels of ACh in the synaptic cleft and consequent regulation of cholinergic activity involved in therapeutic strategies.

\section{Conclusion}

Our results demonstrate that the treatment with E. uniflora extract prevents alterations in metabolic parameters, oxidative damage as well as cholinergic and behavioral changes observed in MetS. Hence, this extract had an antihyperglycemic, antihyperlipidemic and a neuroprotective role since it presented antioxidant and antidepressant-like effects; further studies should be performed on the role of E.uniflora fruit extract in the prevention of MetS. We are currently pursuing this goal.

\section{Conflict of interest}

The authors declare that there is no conflict of interest in the study.

\section{Acknowledgments}

The Brazilian research funding agencies FAPERGS, CAPES and CNPq supported this study. The authors thank Hedy Hofmann for the English review.

\section{References}

[1] S. Ketmanee, K. Upa, S. Weerapon, P. Poungrat, P. Patchareewan, E.G. P.Parichat, K. Stephen, Veerapol, ferulic acid alleviates changes in a rat model of metabolic syndrome induced by high-carbohydrate, high-fat diet, Nutrient 7 (2015) 6446-6464.

[2] N. Gupta, K. Goel, P. Shah, A. Misra, Childhood obesity in developing countries: epidemiology, determinants, and prevention, Endocr. Rev. 33 (2012) 48-70. 
[3] C.G. Souza, J.D. Moreira, I.R. Siqueira, A.G. Pereira, D.K. Rieger, D.O. Souza, T.M. Souza, L.V. Portela, M.L.S. Perry, Highly palatable diet consumption increases protein oxidation in rat frontal cortex and anxiety-like behavior, Life Sci. 81 (2007) 198-203.

[4] K. Boonloh, V. Kukongviriyapan, B. Kongyingyoes, U. Kukongviriyapan, S. Thawornchinsombut, P. Pannangpetch, Rice bran protein hydrolysates improve insulin resistance and decrease pro-inflammatory cytokine gene expression in rats fed a high carbohydrate-High fat diet, Nutrient 7 (2015) 6313-6329.

[5] G. Murdolo, D. Bartolini, C. Tortoioli, M. Piroddi, L. Luliano, F. Galli, Lipokines and oxysterols. Novel adipose-derived lipid hormones linking adipose dysfunction and insulin resistance, Free Radic. Biol. Med. 65 (2013) 811-820.

[6] E. Nizri, Y. Hamra-Amitay, C. Sicsic, I. Lavon, T. Brenner, Anti-inflammatory propertiesof cholinergic up-regulation: a new role for acetylcholinesterase inhibitors, Neuropharmacology 50 (2006) 540-547.

[7] A. Zarros, C. Liapi, P. Galanopoulou, K. Marinou, Z. Mellios, N. Skandali, H. AlHumadi, F. Anifantaki, E. Gkrouzman, S. Tsakiris, Effects of adult-onset streptozotocin-induced diabetes on the rat brain antioxidant status and the activities of acetylcholinesterase, $\left(\mathrm{Na}^{+}, \mathrm{K}^{+}\right)$- and $\mathrm{Mg}^{2+-}$ ATPase: modulation by L-cysteine, Metab. Brain Dis. 24 (2009) 337-348.

[8] J.E. Morley, W.A. Banks, Lipids and cognition, J. Alzheimers Dis. 20 (2010) 737 747.

[9] F.N. Victoria, A.S. Brahm, L. Savegnago, E.J. Lenardão, Involvement of serotoninergic and adrenergic systems on the antidepressant-like effect of E. uniflora L. leaves essential oil and further analysis of its antioxidant activity, Neurosci. Lett. 544 (2013) 105-109.

[10] L. Silva, E. Figueiredo, N. Ricardo, L. Vieira, R. Figueiredo, L. Brasil, C. Gomes Quantification of bioactive compounds in pulps and by-products of tropical fruits from Brazil, Food Chem 143 (2014) 398-404.

[11] H.A. Martinez-Correa, P.M. Magalhães, C.L. Queiroga, C.A. Peixoto, A.L. Oliveira F.A. Cabral, Extracts from pitanga (Eugenia uniflora L.) leaves: influence of extraction process on antioxidant properties and yield of phenolic compounds, J. Supercrit. Fluids 55 (2011) 998-1006.

[12] I.J. Kade, E.O. Ibukun, C.W. Nogueirab, J.B.T. Da Rocha, Sun-drying diminishes the antioxidative potentials of leaves of Eugenia uniflora against formation of thiobarbituric acid reactive substances induced in homogenates of rat brain and liver, Exp. Toxicol. Pathol. 60 (2008) 365-371.

[13] V.L.A.G. Lima, E.A. Mélo, D.E.S. Lima, Fenólicos e carotenoides totais da pitanga, Sci. Agric. 59 (2002) 447-450.

[14] C.L. Bordignon Jr, V. Francescatto, A.A. Nienow, E. Calvete, F.H. Reginatto, Influência do $\mathrm{pH}$ da solução extrativa no teor de antocianinas em frutos de morango, Ciênc. Tecnol. Aliment. 29 (2009) 183-188.

[15] V.L. Singleton, R. Orthefer, R. Lamuela-Ranventós, Analysis of total phenols and other oxidation substrates and antioxidants by means of Folin-Ciocalteau reagent, in: L. Parker (Ed.), Methods Enzymol, Academic Press, 1999, pp. 152 159.

[16] G. Miliauskas, P.R. Venskutonis, T.A. van Beek, Screening of radical scavenging activity of some medicinal and aromatic plant extracts, Food Chem. 85 (2004) 231-237.

[17] J. Lee, R.W. Durst, R.E. Wrolstad, Determination of total monomeric anthocyanin pigment content of fruit juices, beverages, natural colorants and wines by the pH differential method: collaborative study, J. AOAC Int. 8 (2005) 1269-1278.

[18] P.S. Oliveira, M. Gazal, N.P. Flores, A.R. Zimmer, V.C. Chaves, F.H. Reginatto, M.P. Kaster, R.G. Tavares, R.M. Spanevello, C.L. Lencina, F.M. Stefanello, Vaccinium virgatum fruit extract as an important adjuvant in biochemical and behavioral alterations observed in animal model of metabolic syndrome, Biomed. Pharmacother. 88 (2017) 939-947.

[19] M.P. Kaster, J. Budni, R.W. Binfare, A.R. Santos, A.L. Rodrigues, The inhibition of different types of potassium channels underlies the antidepressant-like effect of adenosine in the mouse forced swimming test, Prog. Neuropsychopharmacol. Biol. Psychiatry 31 (2007) 690-696.

[20] M.P. Kaster, A.O. Rosa, M.M. Rosso, E.C. Goulart, A.R. Santos, A.L. Rodrigues, Adenosine administration produces an antidepressant-like effect in mice: evidence for the involvement of A1 and A2A receptors, Neurosci. Lett. 55 (2004) 21-24.

[21] T.N. Huynh, A.M. Krigbaum, J.J. Hanna, C.D. Conrad, Sex differences and phase of light cycle modify chronic stress effects on anxiety and depressive-like behavior, Behav. Brain Res. 222 (2011) 212-222.

[22] O.H. Lowry, N.J. Rosebrough, A.L. Farr, R.J. Randall, Protein measurement with The Folin phenol reagent, J. Biol. Chem. 193 (1951) 265-275.

[23] M.A. Bradford, Rapid and sensitive method for the quantification of microgram quantities of protein utilizing the principle of protein-dye binding, Anal. Biochem. 72 (1976) 248-254.

[24] H. Ohkawa, N. Ohishi, K. Yagi, Assay for lipid peroxides in animal tissues by thiobarbituric acid reaction, Anal. Biochem. 95 (1979) 351-358.
[25] M.Y. Aksenov, W.R. Markesbery, Changes in thiol content and expression of glutathione redox system genes on the hippocampus and cerebellum in Alzheimer's disease, Neurosci. Lett. 302 (2001) 141-145.

[26] H. Aebi, Catalase in vitro, Methods Enzymol. 105 (1984) 121-126.

[27] H.P. Misra, I. Fridovich, The role of superoxide anion in the autoxidation of epinephrine and a simple assay for superoxide dismutase, J. Biol. Chem. 247 (1972) 3170-3175.

[28] G.L. Ellman, K.D. Courtney, V. Andres, R.M. Featherstone, A new and rapid colorimetric determination of acetylcholinesterase activity, Biochem. Pharmacol. 7 (1991) 88-95.

[29] L.S. Einbond, K.A. Reynertson, X.D. Luo, M.J. Basile, E.J. Kennelly, Anthocyanin antioxidants from edible fruits, Food Chem. 84 (2004) 23-28.

[30] D.J. Soares, M. Pignitter, M.M. Ehrnhöfer-Ressler, J. Walker, I.M. Brasil, V. Somoza, Identification and quantification of oxidoselina-1, 3, 7(11)-trien-8one and cyanidin-3-glucoside as one of the major volatile and non-volatile low-molecular-weight constituents in pitanga pulp, Plos One 10 (2015) 1-9.

[31] K. Aschbacher, S. Kornfeld, M. Picard, E. Puterman, J.P. Havel, K. Stanhope, R.H. Lustig, E. Epel, Chronic stress increases vulnerability to diet-related abdominal fat, oxidative stress, and metabolic risk, Psychoneuroendocrinology 46 (2014) $14-22$.

[32] M.J. Amiot, C. Riva, A. Vinet, Effects of dietary polyphenols on metabolic syndrome features in humans: a systematic review, Obes. Rev. 17 (2016) 573586.

[33] P.K. Bagul, H. Middela, S. Matapally, R. Padiya, T. Bastia, K. Madhusudana, B. Raghunath Reddy, Sumana Chakravarty, Sanjay K. Banerjee, Attenuation of insulin resistance, metabolic syndrome and hepatic oxidative stress by resveratrol in fructose-fed rats, Pharmacol. Res. 66 (2012) 260-268.

[34] R. Mopuri, M. Ganjayi, K.S. Banavathy, B.N. Parim, B. Meriga, Evaluation of antiobesity activities of ethanolic extract of Terminalia paniculata bark on high fat diet-induced obese rats, BMC Complement. Altern. Med. 15 (2015) 65-76.

[35] L. Lozano, R. Van der Werf, W. Bietiger, E. Seyfritz, C. Peronet, M. Pinget, N. Jeandidier, E. Maillard, E. Marchioni, S. Sigrist, S. Dal, High-fructose and highfat diet-induced disorders in rats: impact on diabetes risk, hepatic and vascular complications, Nutr. Metab. 15 (2016) 1-13.

[36] A.G.V. Costa, D.F. Garcia-Diaz, P. Jimenez, P.I. Silva, Bioactive compounds and health benefits of exotic tropical red-black berries, J. Funct. Foods 5 (2016) 539-549.

[37] K. Hanhineva, R. Törrönen, I. Bondia-Pons, J. Pekkinen, M. Kolehmainen, H. Mykkänen, K. Poutanen, Impact of dietary polyphenols on carbohydrate metabolism, Int. J. Mol. Sci. 11 (2010) 1365-1402.

[38] M.A. Gammone, G. Riccioni, N. D'orazio, Carotenoids: potential allies of cardiovascular health? Food Nutr. Res. 59 (2015) (267-262).

[39] S. Manzano, G. Williamson, Polyphenols and phenolic acids from strawberry and apple decrease glucose uptake and transport by human intestinal Caco2cells, Mol. Nutr. Food Res. 54 (2010) 1773-1780.

[40] C.L. Hsu, G.C. Yen, Effect of gallic acid on high fat diet-induced dyslipidaemia, hepatosteatosis and oxidative stress in rats, Br. J. Nutr. 98 (2007) 727-735.

[41] T. Tsuda, Regulation of adipocyte function by anthocyanins; possibility of preventing the metabolic syndrome, J. Agric. Food Chem. 56 (2008) 642-646.

[42] S. Treviño, P. Aguilar-Alonso, J.A. Flores Hernandez, E. Brambila, J. Guevara, G. Flores, G. Lopez-Lopez, G. Muñoz-Arenas, J.C. Morales-Medina, V. Toxqui, B. Venegas, A. Diaz, A high calorie diet causes memory loss, metabolic syndrome and oxidative stress into hippocampus and temporal cortex of rats, Synapse 69 (2015) 421-433.

[43] N.S.G. Schumacher, T.C. Colomeu, D. De Figueiredo, V.C. Carvalho, C.B.B. Cazarin, M.A. Prado, L.M.M. Meletti, R.L. Zollner, Identification and antioxidant activity of the extracts of Eugenia uniflora leaves, characterization of the antiInflammatory properties of aqueous extract on diabetes expression in an experimental model of spontaneous type 1 diabetes (NOD mice), Antioxidants 4 (2015) 662-680.

[44] Z. Madani, W.J. Malaisse, D. Ait-Yahia, A comparison between the impact of two types of dietary protein on brain glucose concentrations and oxidative stress in high fructose-induced metabolic syndrome rats, Biomed. Rep. 3 (2015) 731-735.

[45] A. Kuhad, R. Sethi, K. Chopra, Lycopene attenuates diabetes-associated cognitive decline in rats, Life Sci. 83 (2008) 128-134.

[46] A. Pan, N. Keum, O. Okereke, Bidirectional association between depression and metabolic syndrome: a systematic review and meta-anal sis of epidemiological studies, Diabetes Care 35 (2012) 1171-1180.

[47] S. Liu, T. Li, H. Liu, X. Wang, S. Bo, Y. Xie, X. Bai, L. Wu, Z. Wang, D. Liu, Resveratrol exerts antidepressant properties in the chronic unpredictable mild stress model through the regulation of oxidative stress and mTOR pathway in the rat hippocampus and prefrontal cortex, Behav. Brain Res. 302 (2016) 191 199. 